\title{
Probing the Time Variation of a Fine Structure Constant Using Galaxy Clusters and the Quintessence Model
}

\author{
Zhi-E Liu (刘志娥) ${ }^{1}$ (D), Wen-Fei Liu (刘文斐) ${ }^{1}$, Tong-Jie Zhang (张同杰) ${ }^{1,2}$ (D), Zhong-Xu Zhai (翟忠旭) $)^{3}$ (iD), and Kamal Bora ${ }^{4}$ (iD \\ ${ }^{1}$ College of Physics and Electronic Engineering, Qilu Normal University Jinan 250200, People's Republic of China; zhieliu@ 163.com, tjzhang@bnu.edu.cn \\ ${ }^{2}$ Department of Astronomy, Beijing Normal University Beijing 100875, People's Republic of China \\ ${ }^{3}$ IPAC, California Institute of Technology Mail Code 314-6, 1200 E. California Blvd., Pasadena, CA 91125, USA \\ ${ }_{4}^{4}$ Department of Physics, Indian Institute of Technology Hyderabad, Kandi, Telangana-502284, India \\ Received 2021 June 18; revised 2021 August 22; accepted 2021 August 24; published 2021 November 16
}

\begin{abstract}
We explore a possible time variation of the fine structure constant $\left(\alpha \equiv e^{2} / \hbar c\right)$ using the Sunyaev-Zel'dovich effect measurements of galaxy clusters along with their X-ray observations. Specifically, the ratio of the integrated Comptonization parameter $Y_{\mathrm{SZ}} D_{A}^{2}$ and its X-ray counterpart $Y_{X}$ is used as an observable to constrain the bounds on the variation of $\alpha$. Considering the violation of the cosmic distance duality relation, this ratio depends on the fine structure constant of $\sim \alpha^{3}$. We use the quintessence model to provide the origin of $\alpha$ time variation. In order to give a robust test on $\alpha$ variation, two galaxy cluster samples, the 61 clusters provided by the Planck collaboration and the 58 clusters detected by the South Pole Telescope (SPT), are collected for analysis. Their X-ray observations are given by the XMM-Newton survey. Our results give $\zeta=-0.203_{-0.099}^{+0.101}$ for the Planck sample and $\zeta=-0.043_{-0.148}^{+0.165}$ for the SPT sample, indicating that $\alpha$ is constant with redshift within $3 \sigma$ and $1 \sigma$ for the two samples, respectively.
\end{abstract}

Unified Astronomy Thesaurus concepts: Quintessence (1323); Cosmological models (337); Galaxy clusters (584)

\section{Introduction}

The fine structure constant $\alpha \equiv e^{2} / \hbar c$ is at the central position in the system of fundamental physical constants. It measures the strength of the electromagnetic interaction between charged elementary particles in the low-energy limit. Recently, the fine structure constant was determined at an unprecedented precise measurement, that is, $\alpha^{-1}=137.035999206$ with a relative accuracy of 81 parts per trillion (Morel et al. 2020). However, in 1937, Dirac (1937) argued that the fundamental constants of nature may not be pure constants but may vary slowly with the epoch, and he proposed a gravitational constant decreasing proportionally to $t^{-1}$. Since then, some theoretical and experimental investigations allowing spacetime variation of fundamental constants have been put into effect (Uzan 2011, 2003; Martins 2017; Wang \& Chen 2020). In order to remedy the dire consequence (Teller 1948) induced by the varying gravitational constant $G \sim t^{-1}$, Gamow (1967) suggested that $e^{2}$ increases in direct proportion to the age of the universe. Phenomenological models that usually assumed an $\alpha$ varying as some power law or logarithm of time, like those introduced by Gamow, represent the early work of studying varying $\alpha$ (Barrow \& Tipler 1986).

Over the last few decades, the focus has shifted to various extensions of standard models, in which one or more fundamental constants of nature become dynamical quantities. The first self-consistent theory of varying $\alpha$ is the framework constructed by Bekenstein (1982) via modifying Maxwell electrodynamics, which was then extended to a cosmological setting by Sandvik et al. (2002), Barrow et al. (2002b), Barrow $\&$ Mota (2003), and Barrow et al. (2002a), namely the BSBM theory. In the BSBM model, variations in $\alpha$ occur due to a coupling between the electromagnetic field and a massless scalar field in the action. The original BSBM model was then extended by adding a nontrivial potential (Barrow \& Li 2008; Farajollahi \& Salehi 2012), or by allowing the coupling between scalar field $\phi$ and photons as a function of $\phi$
(Barrow \& Lip 2012), or by allowing for both an arbitrary coupling and potential function (Barrow \& Graham 2013). Another class of the extensions of standard models is based on the belief that space has more than three-dimensions, and the extra space dimensions can cause varying fundamental constants (Chodos \& Detweiler 1980; Marciano 1984; Kolb et al. 1986; Barrow 1987). For instance, in Kaluza-Klein (KK) theories, the time evolution of the mean KK radius $R_{\mathrm{KK}}$ of extra spatial dimensions can give rise to time variations of fundamental constants (Marciano 1984).

At present, observational data of $\alpha$ variation are becoming increasingly abundant. The cosmic microwave background (CMB) data (O'Bryan et al. 2015; PLANCK Collaboration 2015; Hart \& Chluba 2018) and the big bang nucleosynthesis (BBN; Iocco et al. 2009; Mosquera \& Civitarese 2013; Clara \& Martins 2020) can constrain $\alpha$ variations in the early universe. The Planck Collaboration (2015) obtained $\Delta \alpha / \alpha \approx 10^{-3}$ at a redshift of $z \approx 10^{3}$ by analyzing the CMB spectra, and the constraints given by the abundance of the light elements emerged during BBN are not very tight (approximately $\Delta \alpha / \alpha<10^{-2}-10^{-3}$ at $z \approx 10^{9}-10^{10}$ ), too (Mosquera \& Civitarese 2013). The 1.8 billionyear-old natural nuclear reactor at the Oklo Uranium Mine in Gabon can give much tighter bounds on the time variations of $\alpha$ $\left(\Delta \alpha / \alpha \approx 10^{-7}-10^{-8}\right.$; Damour \& Dyson 1996; Lamoreaux \& Torgerson 2004). The most sensitive constraints on $\Delta \alpha / \alpha$ were achieved at $z \approx 1-6$ from the spectral lines significantly affected by relativistic effects in absorbing clouds around distant quasars. The quasar absorption lines observed by Keck/High Resolution Echelle Spectrometer (HIRES) and Very Large Telescope (VLT)/ Ultraviolet and Visual Echelle Spectrograph (UVES) telescopes have produced a large data sample that describes the dependence of $\Delta \alpha / \alpha$ on redshift $z$, in which the variations of $\alpha$ are normally constrained by $\Delta \alpha / \alpha \approx 10^{-5}-10^{-6}$ (Murphy et al. 2001a, 2001b, 2001c, 2003, 2004, 2007, 2008; Chand et al. 2004; Srianand et al. 2004; Webb et al. 1999, 2001). In addition, the spatial variations of $\alpha$ observed by comparing the results from 
Keck and VLT are of interest to study. Specifically, at $z>1.8$ the northern sky observations of the Keck telescope suggested a smaller value of the fine structure constant $(\Delta \alpha / \alpha=(-0.74 \pm$ $0.17) \times 10^{-5}$ ), but the southern sky observations of the VLT telescope showed an increasing fine structure constant $\left(\Delta \alpha / \alpha=(0.61 \pm 0.20) \times 10^{-5}\right.$; Webb et al. 2011; King et al. 2012). This could be due to undetected systematic effects, but may also hint to new physics. More recently, a constraint on the relative variation of $\alpha$ below $10^{-5}$ is obtained by comparing the absorption lines of late-type evolved giant stars from the S-star cluster orbiting the supermassive black hole in our Galactic center with the absorption lines in the lab (Hees et al. 2020). The absorption lines of white dwarf stars can also be used to constrain $\Delta \alpha / \alpha$ (Berengut et al. 2013).

Some work consider a class of dilaton runaway models, where $\Delta \alpha / \alpha=-\gamma \ln (1+z)$, and indirectly constrain $\Delta \alpha / \alpha$ by constraining $\gamma$. Most of this kind of work employed the observations of the Sunyaev-Zel'dovich effect (SZ effect, hereafter) combined with observations of the X-ray surface brightness of galaxy clusters, for which the former can be characterized by the integrated Comptonization parameter $Y_{\mathrm{SZ}} D_{A}^{2}$ and the latter by the $Y_{X}$ parameter (Holanda et al. 2017, 2016a, 2016b; Colaço et al. 2019; Bora \& Desai 2021a, 2021b). Alternatively, Colaço et al. (2021a, 2021b) used combined measurements of strong gravitational lensing systems and Type Ia supernovae to constrain $\gamma$. Instead of basing $\alpha$ on runaway models, Galli (2013) assumed that $\alpha$ can linearly vary with redshift, i.e., $\alpha / \alpha_{0}=A_{\text {lin }}(1+z)$, and then studied whether $\alpha$ is time-dependent by constraining $A_{\text {lin }}$ using the SZ effect and its X-ray counterpart of galaxy clusters.

In this paper, we will consider a specific model for the variation of the fine structure constant $\alpha$ driven by a typical quintessence scenario, i.e., a linear coupling with the electromagnetic term in Lagrangian.

\section{Coupling of the Quintessence to the Electromagnetic Field}

Quintessence is a type of dynamical scalar field models (Padmanabhan 2003). It has been used to model the darkenergy component in the universe. We expect the quintessence field to couple with the electromagnetic sector of the matterradiation Lagrangian and induce a time variation of the fine structure constant $\alpha$. The general form of the action involving a quintessence scalar field and its coupling with the electromagnetic term can be written as (Marra \& Rosati 2005)

$$
\begin{aligned}
S= & \frac{1}{16 \pi G} \int d^{4} x \sqrt{-g} R \\
& +\int d^{4} x \sqrt{-g}\left[\frac{1}{2} \partial^{\mu} \phi \partial_{\mu} \phi-V(\phi)\right] \\
- & \frac{1}{4} \int d^{4} x \sqrt{-g} B_{F}(\phi) F_{\mu \nu} F^{\mu \nu}+\int d^{4} x \sqrt{-g} \mathcal{L},
\end{aligned}
$$

where $G$ is Newton's Gravitational constant and $\mathcal{L}$ represents the Lagrangian density including the standard model fields and a hypothetical dark matter sector. $B_{F}(\phi)$ describes the coupling between quintessence and the electromagnetic field and allows for the evolution in $\phi$. The effective fine structure constant depends on the value of $\phi$ as (Copeland et al. 2004)

$$
\alpha(\phi)=\frac{\alpha_{0}}{B_{F}(\phi)},
$$

where $\alpha_{0}$ is the fine structure constant measured today. Therefore, we get the relative variation of $\alpha$ :

$$
\frac{\Delta \alpha}{\alpha} \equiv \frac{\alpha(t)-\alpha_{0}}{\alpha_{0}}=\frac{1-B_{F}(\phi)}{B_{F}(\phi)} .
$$

We consider a homogeneous and isotropic FriedmannRobertson-Walker (FRW) cosmology described by the line element

$$
d s^{2}=-d t^{2}+a^{2}(t)\left[\frac{d r^{2}}{1-K r^{2}}+r^{2}\left(d \theta^{2}+\sin ^{2} \theta d \varphi^{2}\right)\right],
$$

where $a(t)$ and $K$ are the scale factor and spatial curvature, respectively. Combining Equations (1) and (4) can yield equations describing the evolution of the quintessence scalar field in the FRW universe, that is, the Friedmann equation (Park \& Ratra 2019)

$$
\left(\frac{H}{H_{0}}\right)^{2}=\frac{1}{1-\frac{1}{6}\left(\phi^{\prime}\right)^{2}}\left[\Omega_{m} a^{-3}+\Omega_{r} a^{-4}+\Omega_{k} a^{-2}+\frac{V(\phi)}{3 H_{0}^{2}}\right]
$$

and the Klein-Gordon equation (Park \& Ratra 2019)

$$
\phi^{\prime \prime}+\left(3+\frac{\dot{H}}{H^{2}}\right) \phi^{\prime}+\frac{d V(\phi)}{d \phi} \frac{1}{H^{2}}=0,
$$

where $\phi^{\prime} \equiv d \phi / d \ln a$, and an overdot denotes the time derivative $d / d t$. We have set the present scale factor to $a_{0}=1$ and chose the units such that the Newtonian gravitational constant $G \equiv 1 / 8 \pi . H=\dot{a} / a$ is the Hubble parameter and $H_{0}$ is the Hubble constant. The present value of the nonrelativistic matter density parameter $\Omega_{m}$ is the sum of the present baryonic matter and the cold dark matter (CDM) density parameters, $\Omega_{m}=\Omega_{b}+\Omega_{c}$. $\Omega_{r}$ is the present value of the radiation density parameter, and $\Omega_{k}$ is the present value of the spatial curvature density parameter. We assume a spatially flat universe, $\Omega_{k}=0$, and set $\Omega_{m}=0.315 \pm 0.007, H_{0}=67.4 \pm$ $0.5 \mathrm{kms}^{-1} \mathrm{Mpc}^{-1}$ according to the Planck 2018 results (Chen et al. 2019; Planck Collaboration 2020). $\Omega_{r}$ is not a free parameter but is determined by $\Omega_{r}=\Omega_{m} a_{\mathrm{eq}}$, where $a_{\mathrm{eq}}$ is the scale factor at the epoch of matter-radiation equality given by $a_{\mathrm{eq}}=\frac{4.15 \times 10^{-5}}{\Omega_{m} h^{2}}$ (Dodelson \& Schmidt 2020). Here $h=H_{0} /\left(100 \mathrm{~km} \mathrm{~s}^{-1} \mathrm{Mpc}^{-1}\right)$.

A general functional form of the quintessence potential $V(\phi)$ can be written as a combination of a power law and exponential functions. Copeland et al. (2004) discussed in detail the effect induced by different quintessence models on the cosmological $\Delta \alpha$. In our work, we only consider an inverse power-law potential (Peebles \& Ratra 1988; Samushia \& Ratra 2006, 2009; Chen \& Ratra 2011; Park \& Ratra 2019),

$$
V(\phi)=V_{1} \phi^{-n},
$$

where $V_{1}$ and $n$ are non-negative constant parameters. In the limit $n=0$ the quintessence dark energy becomes the cosmological constant $\Lambda$. The exponent parameter $n$ has been constrained to $[0,6]$ by various observational data (Park \& Ratra 2019). Marra \& Rosati (2005) showed that choosing different quintessence potentials gives a subdominant effect on 
the cosmological variation of $\alpha$ with respect to changing the coupling function $B_{F}(\phi)$. Our tests also show that different values of $n$ only have a mild impact on the result, and fixing this parameter can improve the efficiency of the computation significantly. Thus, following Marra \& Rosati (2005) we set $n=1.0$ in order to have the correct attractor equation of state (Marra \& Rosati 2005). By definition, the pressure $p_{\phi}$ and energy density $\rho_{\phi}$ of the scalar field are given by

$$
\begin{aligned}
& p_{\phi}=\frac{\dot{\phi}^{2}}{2}-V(\phi), \\
& \rho_{\phi}=\frac{\dot{\phi}^{2}}{2}+V(\phi),
\end{aligned}
$$

and the equation of state for the scalar field is $\omega_{\phi}=p_{\phi} / \rho_{\phi}$. The coefficient parameter $V_{1}$ of the potential of Equation (7) can be numerically calculated by making the current energy density $\rho_{\phi}^{(0)}$, which is evolved from Equation (6), equal to

$$
\rho_{c r} \Omega_{\phi}=\rho_{c r}\left(1-\Omega_{m}-\Omega_{r}-\Omega_{k}\right),
$$

where $\rho_{c r}=3 H_{0}^{2}$ is the critical density. Thus, $V_{1}$ is in fact determined by $n$ implicitly. Accordingly, the current darkenergy density parameter is $\Omega_{\phi}=\rho_{\phi}^{(0)} / \rho_{c r}=\left(\phi^{\prime}{ }_{0}\right)^{2} / 6+$ $V\left(\phi_{0}\right) /\left(3 H_{0}^{2}\right)$, where $\phi_{0}$ and $\phi_{0}^{\prime}$ are the current values of $\phi$ and $\phi^{\prime}$.

We numerically solve the cosmological Equations (5)-(6) and then produce the resulting $\Delta \alpha$ at a series of redshifts. We use the initial conditions (Peebles \& Ratra 1988)

$$
\phi=\left[\frac{2}{3} n(n+2)\right]^{1 / 2}\left(\frac{a}{a_{1}}\right)^{3 /(n+2)}
$$

at a scale factor of $a_{i}=10^{-10}$. Here $a_{1}$ denotes the characteristic epoch at which the dominant mass density switches from ordinary matter to the $\phi$ field.

Equation (3) shows that the evolution of $\alpha$ depends on the evolution of $\phi$ via a concrete functional form of $B_{F}(\phi)$, so what follows is the choice of $B_{F}(\phi)$. In principle, there are no constraints on the form of $B_{F}(\phi)$. Marra \& Rosati (2005) proposed a general form of the function $B_{F}(\phi)$, which is characterized by a set of four parameters, and comprehensively discussed various $B_{F}(\phi)$ cases that gave different $\alpha$ histories. In this work we adopt the simplest case originally proposed by Bekenstein (1982), i.e., a linear dependence on $\phi$ such that

$$
B_{F}(\phi)=1-\zeta\left(\phi-\phi_{0}\right),
$$

where $\phi_{0}$ denotes the present quintessence, and $\zeta$ is a parameter describing the strength of the coupling between quintessence and the electromagnetic field, which is to be determined by the observational data. The case $\zeta=0$, or $B_{F}(\phi)=1$, means there is no coupling to the electromagnetic field, and thus the fine structure constant $\alpha$ really remains as a constant during the lifetime of the universe. Substituting Equation (11) into Equation (3) results in

$$
\frac{\Delta \alpha}{\alpha}=\zeta\left(\phi-\phi_{0}\right)
$$

\section{3. $Y_{\mathrm{SZ}}-Y_{X}$ Relation and $\alpha$}

The Sunyaev-Zel'dovich effect $\left(Y_{\mathrm{SZ}}\right)$ and X-ray surface brightness $\left(Y_{X}\right)$ are two observable quantities of galaxy clusters. Since $Y_{\mathrm{SZ}}$ and $Y_{X}$ both reflect the thermal energy of the cluster and are proportional to the total cluster mass, their ratio $Y_{\mathrm{SZ}} D_{A}^{2} / Y_{X}$ should be a constant independent of redshift. This ratio has been used to explore the variation of $\alpha$ (Galli 2013; Holanda et al. 2016b; Colaço et al. 2019; Bora \& Desai 2021a, 2021b), which is also our main focus in this paper.

The high-energy electrons in the ionized intergalactic gas of galaxy clusters can scatter the $\mathrm{CMB}$ photons, via inverse Compton effect, resulting in the distortion of the CMB spectrum. This is the known SZ effect (Sunyaev \& Zel'dovich 1972). The CMB spectral distortion caused by the $\mathrm{SZ}$ effect is proportional to the Compton parameter $y$, which is expressed as

$$
y=\frac{\sigma_{T} k_{B}}{m_{e} c^{2}} \int n_{e} T d l=\frac{\sigma_{T}}{m_{e} c^{2}} \int P d l,
$$

where $k_{B}$ is the Boltzmann constant, $c$ is the speed of light, $m_{e}$ is the electron mass, $n_{e}$ is the number density of electrons, $T$ is the electron temperature, and $P=n_{e} k_{B} T$ is the pressure of the intracluster medium under the assumption of an ideal gas equation of state. Therefore, the Compton parameter quantifies the gas pressure of the intracluster medium integrated along the line of sight. The Thompson cross-section $\sigma_{T}$ can be computed using Feynman diagrams and linked to the fine structure constant $\alpha$ by

$$
\sigma_{T}=\frac{8 \pi}{3}\left(\frac{e^{2}}{m_{e} c^{2}}\right)^{2}=\frac{8 \pi}{3}\left(\frac{\hbar^{2} \alpha^{2}}{m_{e}^{2} c^{2}}\right) .
$$

Integrating the Compton parameter $y$ over the plane perpendicular to the line of sight can provide the spherical integrated Compton parameter, defined as

$$
\begin{aligned}
& Y_{\mathrm{SZ}} D_{A}^{2}=\int y d A \\
& \quad=\frac{\sigma_{T} k_{B}}{m_{e} c^{2}} \int n_{e} T d l d A=\frac{\sigma_{T}}{m_{e} c^{2}} \int P d V .
\end{aligned}
$$

Thus, we can see that $Y_{\mathrm{SZ}} D_{A}^{2}$ depends on the fine structure constant through the Thompson cross-section (see Equation (14)) as (Galli 2013)

$$
Y_{\mathrm{SZ}} D_{A}^{2} \propto \alpha^{2} \text {. }
$$

According to Equation (2), we have

$$
Y_{\mathrm{SZ}} D_{A}^{2} \propto B_{F}^{-2}(\phi) .
$$

The intergalactic hot gas emits mainly through thermal bremsstrahlung. The magnitude of the X-ray emission is quantified by the $Y_{X}$ parameter, which can be acquired by X-ray surface brightness observations, and is defined as (Kravtsov et al. 2006)

$$
Y_{X}=M_{g}(R) T_{X}
$$

where $M_{g}(R)$ is the X-ray determined gas mass within the radius $R$ and $T_{X}$ is the X-ray temperature of the cluster. It has been shown that $M_{g}(R)$ can be written as (Goncalves et al. 2012; 


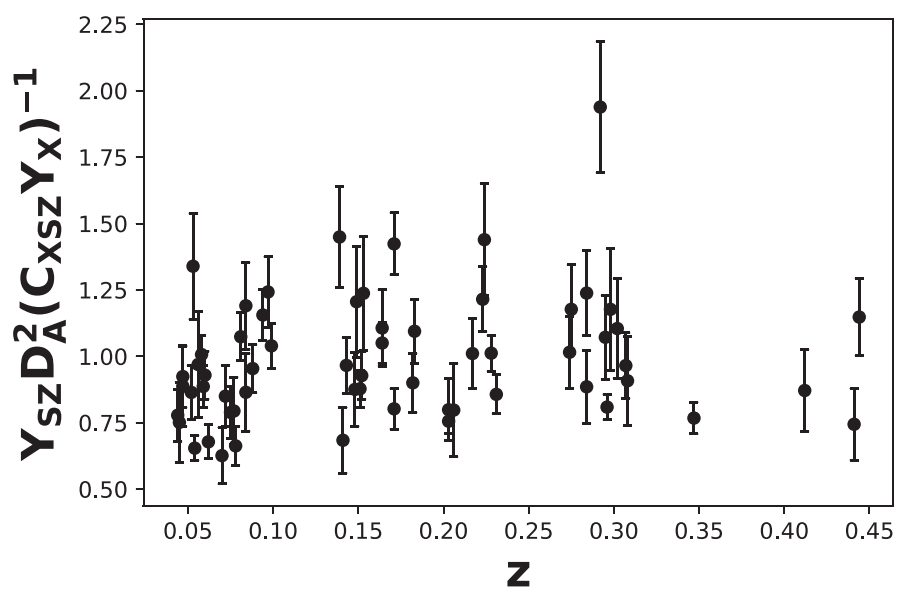

(a)

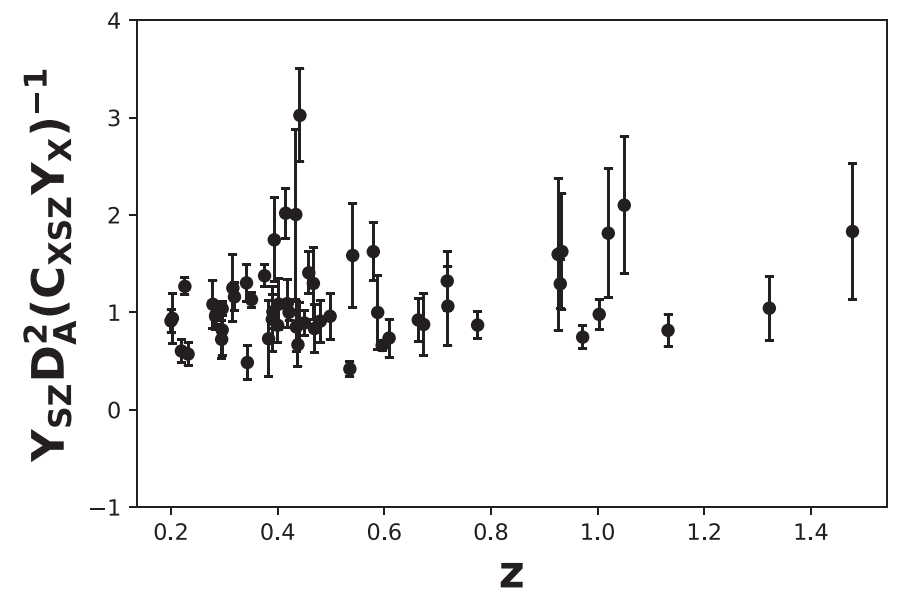

(b)

Figure 1. The observed ratio $Y_{\mathrm{SZ}} D_{A}^{2} / Y_{X} C_{\mathrm{XSZ}}$. They are calculated from (a) the Planck galaxy cluster sample and (b) the SPT galaxy cluster sample.

Colaço et al. 2019)

$$
M_{g}(<\theta) \propto \alpha^{-3 / 2} D_{L} D_{A}^{3 / 2},
$$

where $D_{L}$ is the luminosity distance. This equation shows that $M_{g}(R)$ depends not only on the fine structure constant but also on the validity of the cosmic distance duality relation (CDDR), $D_{L}=(1+z)^{2} D_{A}$. A variation of $\alpha$ will lead to a violation of the CDDR (Hees et al. 2014), which is normally described as $D_{L}=\eta(z)(1+z)^{2} D_{A}$. Consequently, $Y_{X}$ will scale to $\alpha(z)$ and $\eta(z)$ by

$$
Y_{X} \propto M_{g}(<\theta) \propto \alpha(z)^{-3 / 2} \eta(z)
$$

According to the scalar field theory, $\alpha(z)$ and $\eta(z)$ are linked by (Hees et al. 2014)

$$
\frac{\Delta \alpha}{\alpha} \equiv \frac{\alpha(z)-\alpha_{0}}{\alpha_{0}}=\eta(z)^{2}-1 .
$$

Together with Equation (2) we have

$$
Y_{X} \propto \alpha^{-1} \propto B_{F}(\phi) .
$$

Then, based on Equations (17) and (22), the dimensionless ratio of $Y_{\mathrm{SZ}} D_{A}^{2}$ to $Y_{X}$ can be related to the coupling strength $B_{F}(\phi)$ by

$$
\frac{Y_{\mathrm{SZ}} D_{A}^{2}}{Y_{X}} \propto B_{F}(\phi)^{-3} .
$$

This ratio can also been expressed in the following form (Galli 2013):

$$
\frac{Y_{\mathrm{SZ}} D_{A}^{2}}{Y_{X}} \propto C_{\mathrm{XSZ}} \frac{\int n_{e}(r) T(r) d V}{T(R) \int n_{e}(r) d V},
$$

where

$$
C_{\mathrm{XSZ}}=\frac{\sigma_{T}}{m_{e} c^{2}} \frac{1}{\mu_{e} m_{p}} \approx 1.416 \times 10^{-19}\left(\frac{\mathrm{Mpc}^{2}}{M_{\odot} \mathrm{keV}}\right) .
$$

The numerator and denominator in Equation (24) are both approximations of the thermal energy of the cluster. As discussed in Colaço et al. (2019), this ratio would be exactly constant with redshift if no new physics are assumed. As done by Galli (2013), Colaço et al. (2019), and Bora \& Desai (2021a), and considering Equation (11), we can rewrite the ratio in Equation (23) into

$$
\frac{Y_{\mathrm{SZ}} D_{A}^{2}}{Y_{X} C_{\mathrm{XSZ}}}=\mathrm{CB}_{F}(\phi)^{-3}=C\left(1-\zeta\left(\phi-\phi_{0}\right)\right)^{-3},
$$

where $C$ is a constant to be determined. The case of $C \simeq 1$ indicates that the galaxy clusters used in this analysis are isothermal.

We are interested in a possible $\alpha$ variation as predicted by the quintessence model. The quintessence scalar field $\phi$ evolves via Equations (5) and (6), then the constants $C$ and $\zeta$ can be determined by observational measurements. As shown in Equation (12), if the resulting $\zeta$ departs from zero significantly, a time variation for $\alpha$ should be established.

\section{Galaxy Cluster Samples}

Large solid angle surveys employing the SZ effect have been carried out with the South Pole Telescope (SPT; Carlstrom et al. 2011), Planck (Ade et al. 2011a), and the Atacama Cosmology Telescope (Fowler et al. 2007). One data set used here is the SZ and X-ray data extracted from Table 1 of Ade et al. (2011a). The SZ effect measurements in the direction of 62 nearby galaxy clusters $(z<0.5)$, which is a subsample of the Planck Early Sunyaev-Zel'dovich (ESZ) cluster sample (Ade et al. 2011b), were detected at a high signal-to-noise ratio $(S / N \geqslant 6)$ in the first Planck all-sky data set. These galaxy clusters are not contaminated by flares and their morphologies are regular enough that spherical symmetry can be assumed. They had also been observed by the XMM-Newton telescope, and their $Y_{X}$ measurements were obtained with the deep XMMNewton X-ray data (Piffaretti et al. 2011). As done by Galli (2013), cluster A2034 is excluded from the analysis since its redshift estimate is discordant in Ade et al. (2011a) and Mantz et al. (2010). So we use 61 Planck ESZ clusters in this analysis covering the redshift range of $0.044<z<0.44$ (see Figure 1(a)). The $Y_{\mathrm{SZ}}$ and $Y_{X}$ parameters are labeled as $D_{A}^{2} Y_{500}$ and $Y_{X, 500}$, respectively. Here 500 indicates a radius $R_{500}$ at which the mean matter density of the cluster is 500 times that of the critical density of the universe at the redshift of the cluster. The computation of the $Y_{\mathrm{SZ}}$ parameter requires shaping the thermal pressure $(P)$ of the intracluster medium for 


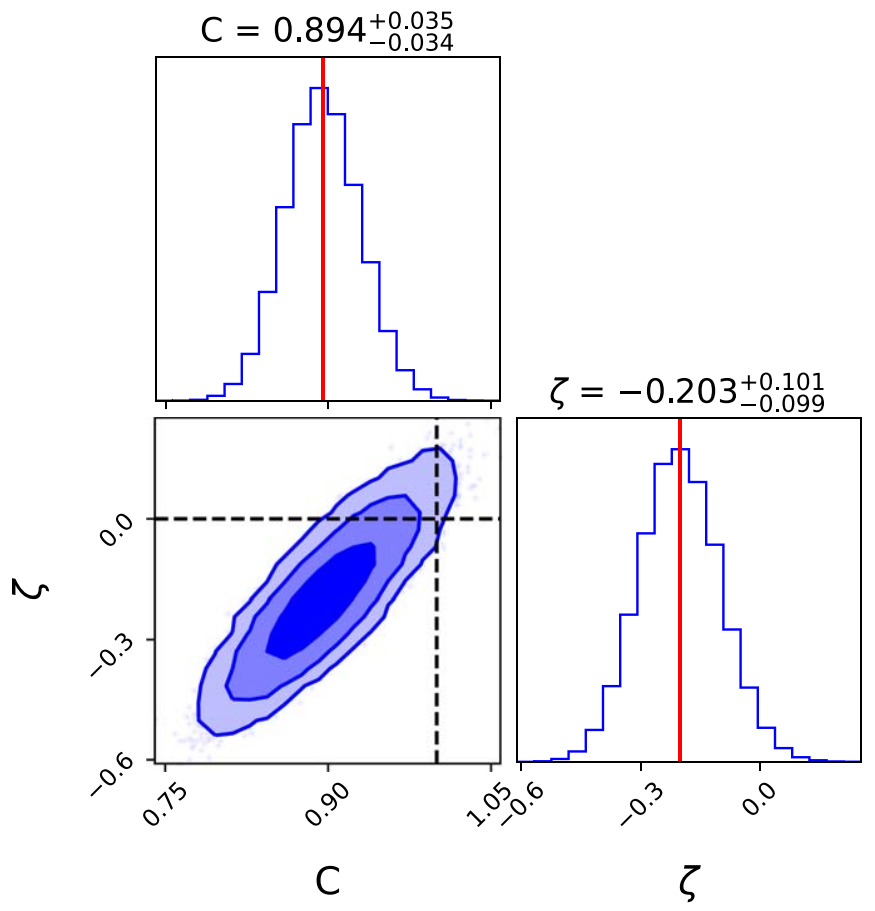

Figure 2. Contours of $1 \sigma, 2 \sigma$, and $3 \sigma$ on the $\zeta-C$ plane and the corresponding one-dimensional marginalized likelihood distributions for the Planck clusters.

each galaxy cluster by using the universal pressure profile (Arnaud et al. 2010). Furthermore, the X-ray temperatures are defined within a cylindrical annulus of radius $[0.15-0.75] R_{500}$. See Galli (2013) and Colaço et al. (2019) for more comments on the data set.

Another data set used in our analysis is the SPT galaxy cluster sample. SPT is a $10 \mathrm{~m}$ telescope at the South Pole that images the sky at three different frequencies, i.e., $95 \mathrm{GHz}$, $150 \mathrm{GHz}$, and $220 \mathrm{GHz}$ (Carlstrom et al. 2011). SPT has detected 516 galaxy clusters via the SZ effect in the 2500 square degree SPT-SZ survey at $0<z<1.8$ with masses $M_{500} \geqslant 3 \times 10^{14} M_{\odot}$, and approximately $20 \%$ of the sample lies at $z>0.8$ (Bleem et al. 2015). The redshifts of the SPT cluster candidates have been obtained through dedicated optical surveys and follow-up programs (Desai et al. 2012; Song et al. 2012; Saro et al. 2015). Their properties were described in Bleem et al. (2015), among which the redshift values were updated in Bocquet et al. (2019). The $Y_{\text {SZ }}$ value of each SPT cluster is measured through integrating the thermal SZ signal in a cylindrical volume within a 0.75 -radius aperture. Note their $\mathrm{X}$-ray counterpart, $Y_{X}$, is measured within a three-dimensional sphere with a radius of $R_{500}$. In order to compare $Y_{\mathrm{SZ}}$ with $Y_{X}$, one needs to convert the SPT $Y_{\mathrm{SZ}}$ values to spherically integrated $Y_{\mathrm{SZ}}$ within the same radius at which $Y_{X}$ was measured. This conversion is performed based on the following expressions (Arnaud et al. 2010):

$$
\begin{gathered}
Y_{\mathrm{cyl}}\left(R_{1}\right)=Y_{\mathrm{sph}}\left(R_{b}\right)-\frac{\sigma_{T}}{m_{e} c^{2}} \int_{R_{1}}^{R_{b}} 4 \pi P(r) \sqrt{r^{2}-R_{1}^{2}} r d r \\
Y_{\mathrm{sph}}\left(R_{2}\right)=\frac{\sigma_{T}}{m_{e} c^{2}} \int_{0}^{R_{2}} 4 \pi P(r) r^{2} d r .
\end{gathered}
$$

Here, $Y_{\text {cyl }}\left(R_{1}\right)$ is the $Y_{\mathrm{SZ}}$ parameter measured within a cylindrical aperture of radius $R_{1}$, and $Y_{\mathrm{sph}}\left(R_{2}\right)$ is the $Y_{\mathrm{SZ}}$ parameter integrated within a spherical volume of radius $R_{2} . R_{b}$ is the radial extent of the cluster, and $P(r)$ is the gas pressure in the intracluster medium. An analytical form of $P(r)$ is needed to calculate the integrations in Equations (27-28), for which we adopt the universal pressure profile suggested by Arnaud et al. (2010). Following Bora \& Desai (2021a), we set $R_{b}=10 R_{500}$. Since the SPT observation gave $Y_{\mathrm{SZ}}$ values in terms of an aperture of $0.75, R_{1}=0.75 D_{A}$ is required in Equation (27). We put $R_{2}=R_{500}$ in Equation (28), concordant with the radius at which the SPT $Y_{X}$ is measured. In order to be less dependent on the free parameters involved in the universal pressure profile of $P(r)$ and make the computation more reliable, instead of calculating $Y_{\mathrm{sph}}\left(R_{500}\right)$ directly by Equation (28), we estimate $Y_{\mathrm{sph}}\left(R_{500}\right)$ via calculating the ratio of $Y_{\text {sph }}\left(R_{500}\right)$ to $Y_{\text {cyl }}\left(0.75 D_{A}\right)$ from Equations (27) and (28).

The XMM-Newton X-ray observations of 73 of these SZ effect selected clusters have been performed by SPT collaboration members or various non-SPT small programs, among which 15 clusters were excluded due to their low data quality or low redshift $(z<0.2)$. Thus, a sample of 58 clusters with a redshift range of $0.2<z<1.5$ is used for analysis (see Figure 1(b)). The sample has a median mass and redshift of $M_{500}=4.77 \times 10^{14} M_{\odot}$ and $z_{\text {med }}=0.45$, respectively, and five clusters lie at $z>1$. The X-ray observable-mass scaling relations of these clusters were studied in Bulbul et al. (2019), and the details of their XMM-Newton observations, especially $Y_{X}$ values, can be found in Table 1 of Bulbul et al. (2019). Same as the Planck counterparts, the X-ray observables of the sample were measured at $R_{500}$.

\section{Analysis and Results}

In order to constrain the parameters of Equation (26), we minimize the following negative log-likelihood function:

$$
\begin{aligned}
&-2 \ln L=\sum_{i=1}^{N} \ln 2 \pi \sigma_{i}^{2} \\
&+\sum_{i=1}^{N} \frac{\left[R_{\mathrm{obs}, i}-C\left(1-\zeta\left(\phi-\phi_{0}\right)\right)^{-3}\right]^{2}}{\sigma_{i}^{2}},
\end{aligned}
$$

where

$$
R_{\mathrm{obs}, i}=\frac{\left(Y_{\mathrm{SZ}} D_{A}^{2}\right)_{i}}{Y_{X, i} C_{\mathrm{XSZ}}}
$$

denotes the observed values of the ratio in Equation (26) for galaxy clusters, $N$ is the total number of clusters, and $\sigma_{i}$ is the total uncertainty inherited from the observations $\left(Y_{\mathrm{SZ}} D_{A}^{2}\right)_{i}$ and $Y_{X, i}$, plus an intrinsic scatter term $\sigma_{\text {int }}$, that is

$$
\sigma_{i}^{2}=\left(\frac{\sigma_{\mathrm{SZ}, i}}{Y_{X, i} C_{\mathrm{XSZ}}}\right)^{2}+\left(\frac{\left(Y_{\mathrm{SZ}} D_{A}^{2}\right)_{i} \sigma_{X, i}}{Y_{X, i} C_{\mathrm{XSZ}}}\right)^{2}+\sigma_{\mathrm{int}}^{2} .
$$

where $\sigma_{S Z, i}$ and $\sigma_{X, i}$ denote the uncertainty of $\left(Y_{\mathrm{SZ}} D_{A}^{2}\right)_{i}$ and $Y_{X, i}$, respectively. The advantage of using the log-likelihood against the commonly used $\chi^{2}$ criterion is that the log-likelihood function can accommodate the optimization of the intrinsic uncertainty. Thus, for the SPT sample the parameter space to be optimized is $\left\{C, \zeta, \sigma_{\text {int }}\right\}$. For the Planck sample, we followed Galli (2013) and set $\sigma_{\text {int }}=0.17$. So the parameter space of the Planck sample is reduced to $\{C, \zeta\}$, and the objective function of Equation (29) becomes equivalent to the $\chi^{2}$ criterion. Our experiment shows different cosmological parameters only have 


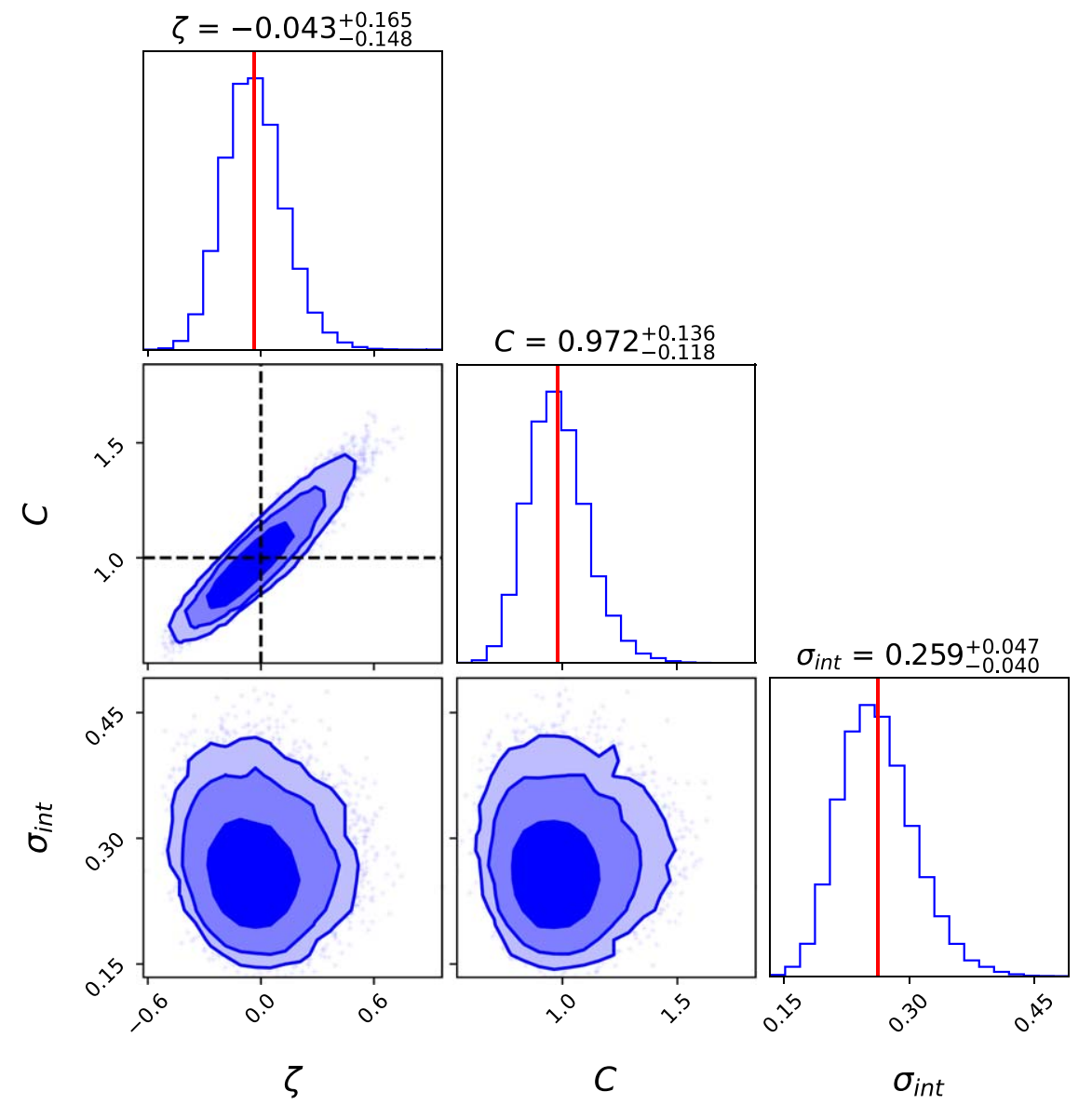

Figure 3. Contours of $1 \sigma, 2 \sigma$, and $3 \sigma$ on the two-dimensional parameter planes and the corresponding one-dimensional marginalized likelihood distributions for the SPT clusters. The red line represents the mean value of each parameter. The dashed lines in the $\zeta-C$ plane mark $\zeta=0$ and $C=1$, which represent $\alpha=\alpha_{0}$ and the isothermality assumption, respectively.

a marginal effect on the result, so we fix the cosmological parameters as $H_{0}=67.4, \Omega_{m}=0.315$ in the implementation of the quintessence model.

Unlike the Planck cluster sample for which the product of $Y_{\mathrm{SZ}}$ and $D_{A}^{2}$, i.e., $Y_{\mathrm{SZ}} D_{A}^{2}$, is already given as an observational quantity, for SPT data we need to estimate the angular diameter distance to each galaxy cluster via

$$
D_{A}=\frac{1}{1+z} \int_{0}^{z} \frac{c d z^{\prime}}{H\left(z^{\prime}\right)}
$$

while $H(z)$ is calculated by the quintessence model (i.e., Equations (5) and (6)).

We maximize the likelihood using the emcee Markov Chain Monte Carlo (MCMC) sampler (Foreman-Mackey et al. 2013). The results on the Planck cluster sample and those on the SPT cluster sample are given in Figures 2 and 3, respectively, which display the $68 \%, 95 \%$, and $99 \%$ confidence level plots along with the marginalized one-dimensional distribution of each parameter. We obtain $\zeta=-0.203_{-0.099}^{+0.101}, C=0.894_{-0.034}^{+0.035}$ from the Planck sample and $\zeta=-0.043_{-0.148}^{+0.165}, C=0.972_{-0.118}^{+0.136}$, and $\sigma_{\text {int }}=0.259_{-0.040}^{+0.047}$ from the SPT sample. We find from Figure 2 that the point $(\zeta=0, C=1)$ is located within the $3 \sigma$ contour for the Planck sample. Therefore, our results indicate that there is no significant evidence for nonzero $\zeta$, implying no time variation in $\alpha$. Furthermore, since $C=1$ holds within $3 \sigma$ and $1 \sigma$ for the Planck sample and the SPT sample, respectively, we conclude that the isothermality assumption of the temperature profile is applicable for both the Planck cluster sample and the SPT cluster sample. It seems that the intrinsic uncertainty of the SPT data $\left(\sigma_{\text {int }}=0.259\right)$ is heavier than that of the Planck data $\left(\sigma_{\text {int }}=0.17\right)$. Considering they stem from different analysis methods, this conclusion needs further validation.

\section{Conclusions}

We explore the possible time variation of the fine structure constant $\alpha$ based on the scaling relation $Y_{\mathrm{SZ}} D_{A}^{2} / C_{X Y Z} Y_{X}$ calculated from galaxy cluster measurements. Following Colaço et al. (2019), this ratio depends on the fine structure constant through the Thompson cross-section and is also affected by the violation of CDDR through the cluster gas mass. Instead of the frequently used runaway dilaton models, we use the quintessence model to provide the theoretical mechanism of generating time-varying $\alpha$. Different from the runaway dilaton model, for which the redshift dependence of the dilaton field is explicitly approximated by $\phi(z)=1-$ $\gamma \ln (1+z)$ at low and intermediate redshifts, the dynamics of the quintessence scalar field is given by an evolution procedure described using Equations (5) and (6). The resulting equality $Y_{\mathrm{SZ}} D_{A}^{2} / Y_{X} C_{\mathrm{XSZ}}=C\left(1-\zeta\left(\phi-\phi_{0}\right)\right)^{-3}$ is derived to link the quintessence field with the cluster observations.

We use the data of two cluster samples to constrain the variation of the fine structure constant. One sample is the observations of 61 galaxy clusters reported by the Planck 


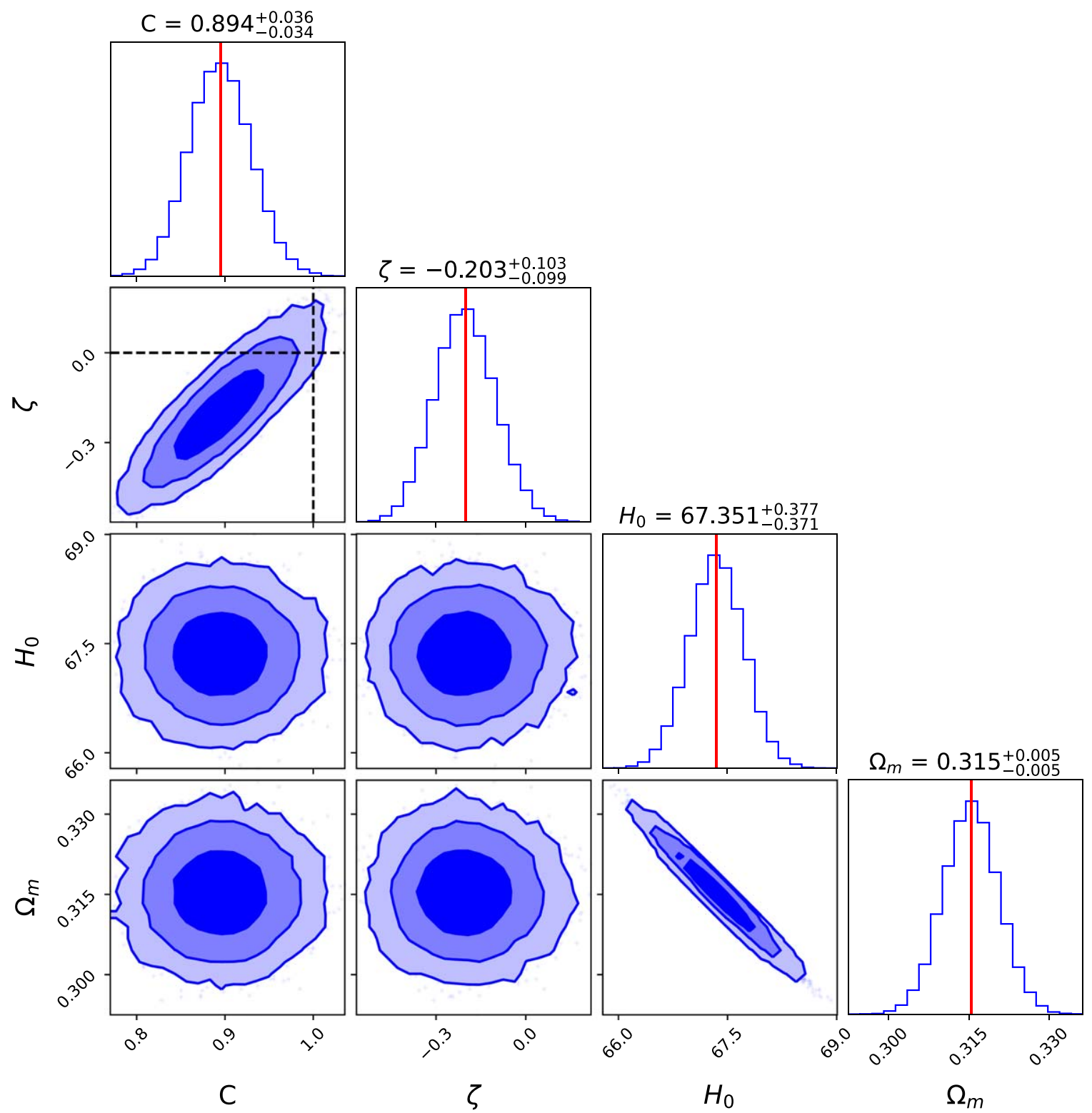

Figure 4. Contours of $1 \sigma, 2 \sigma$, and $3 \sigma$ on the two parameter planes and the corresponding one-dimensional marginalized likelihood distributions for the Planck clusters.

collaboration, and another is the 58 galaxy clusters selected by the SPT-SZ observation. The X-ray counterpart (i.e., $Y_{X}$ ) of both the samples has been observed by the XMM-Newton observations. We give different treatments to the intrinsic uncertainty from the two data sets. For the Planck sample, we set $\sigma_{\text {int }}=0.17$ directly, while for the SPT sample $\sigma_{\text {int }}$ is taken as a free parameter and the determination of its best value is fused in the optimization procedure. In this way, the intrinsic uncertainty $\sigma_{\text {int }}$ for the SPT sample depends on the dispersion of angular diameter distance $D_{A}$ and thus is affected by the uncertainties of the cosmological parameters (i.e., $H_{0}$ and $\Omega_{m}$ ) indirectly. Our analyses show no significant evidence for the fine structure constant $\alpha$ varying with redshift, consistent with previous galaxy cluster-based results (Galli 2013; Colaço et al. 2019; Bora \& Desai 2021a, 2021b). The constant $C$ was found to approach unity sufficiently, indicating that the isothermal temperature profile is universal for describing galaxy clusters.

The number of galaxy clusters available is mainly limited by current X-ray observations. For example, among the 516 clusters detected by the SPT-SZ survey, only 73 clusters have corresponding XMM-Newton X-ray observations. The eROSITA (extended Roentgen Survey with an Imaging Telescope Array) satellite, which was launched in 2019, will perform the first imaging all-sky survey in the medium-energy X-ray range and detect 50,000-100,000 galaxy clusters (Hofmann et al. 2017). More detailed and richer observations in the future may let us check whether the conclusion drawn in this paper is still valid in different redshift domains or not. We expect that the problem of $\alpha$ variation would have a better solution by enlarging the galaxy cluster samples.

We thank the anonymous referee for the helpful and constructive feedback. This work was supported by the National Key R \& D Program of China (2017YFA0402600), the Natural Science Foundation of Shandong Province, China (Grant NO.ZR2019MA059), and the National Natural Science Foundation of China (grant No. 11929301). Z.Z. is supported in part by NASA grant 15-WFIRST15-0008, 


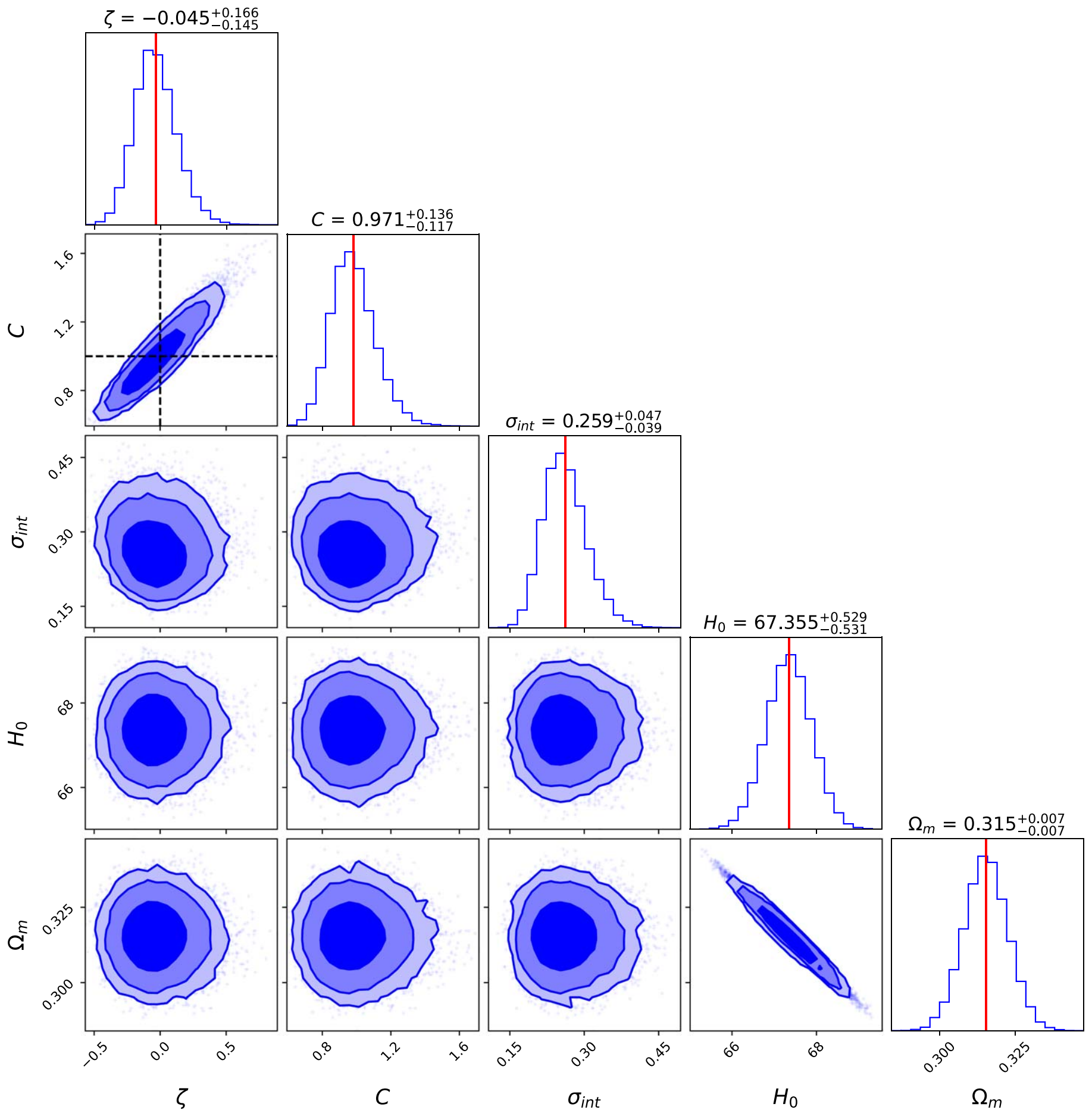

Figure 5. Contours of $1 \sigma, 2 \sigma$, and $3 \sigma$ on the two parameter planes and the corresponding one-dimensional marginalized likelihood distributions for the SPT clusters.

Cosmology with the High Latitude Survey Roman Science Investigation Team (SIT). K.B. acknowledges Department of Science and Technology, Government of India for providing the financial support under the DST-INSPIRE Fellowship program.

\section{Appendix}

To demonstrate that the constraints on the time variation of $\alpha$ are insensitive to the cosmological parameters, we repeat the analysis for both data sets treating $H_{0}$ and $\Omega_{m}$ as free parameters. We assume Gaussian bivariate prior distributions on $H_{0}$ and $\Omega_{m}$ with the following expression

$$
\begin{aligned}
& p^{\text {priors }}(x)=\frac{1}{2 \pi \sqrt{|\Sigma|}} \\
& \quad \times \exp \left(-\frac{1}{2}(x-d) \Sigma^{-1}(x-d)\right),
\end{aligned}
$$

where $x=\left\{H_{0}, \Omega_{m}\right\}$, and their mean values $d=\{67.36,0.3153\}$ and covariance matrix $\Sigma=\left(\begin{array}{cc}0.287 & -3.89 \times 10^{-3} \\ -3.89 \times 10^{-3} & 5.37 \times 10^{-5}\end{array}\right)$ are 
derived from the CosmoMC chains of Planck 2018 TT,TE,EE + lowE+lensing (Chen et al. 2019). We get $C=0.894_{-0.034}^{+0.036}$ and $\zeta=-0.202_{-0.101}^{+0.103}$ for the Planck clusters (see Figure 4) and $\zeta=-0.045_{-0.145}^{+0.166}, C=0.971_{-0.117}^{+0.136}$, and $\sigma_{\text {int }}=0.259_{-0.039}^{+0.047}$ for the SPT clusters (see Figure 5). One can see that compared to the results obtained with fixed $H_{0}$ and $\Omega_{m}$, the difference between the both analyses is insignificant.

\section{ORCID iDs}

Zhi-E Liu (刘志娥) (10 https://orcid.org/0000-0003-3153-1296 Tong-Jie Zhang (张同杰) (10 https://orcid.org/0000-0002-1596$529 \mathrm{X}$

Zhong-Xu Zhai (翟忠旭) (10) https://orcid.org/0000-0001-79845476

Kamal Bora (ib) https://orcid.org/0000-0002-6372-9363

\section{References}

Ade, P. A. R., Aghanim, N., Planck Collaboration, et al. 2011a, A\&A, 536, A8 Ade, P. A. R., Aghanim, N., Planck Collaboration, et al. 2011b, A\&A, 536, A11

Arnaud, M., Pratt, G., Piffaretti, R., et al. 2010, A\&A, 517, A92

Barrow, J. D. 1987, PhRvD, 35, 1805

Barrow, J. D., \& Graham, A. A. H. 2013, PhRvD, 88, 103513

Barrow, J. D., \& Li, B. 2008, PhRvD, 78, 083536

Barrow, J. D., \& Lip, S. Z. W. 2012, PhRvD, 85, 023514

Barrow, J. D., Magueijo, J., \& Sandvik, H. B. 2002a, PhRvD, 66, 043515

Barrow, J. D., \& Mota, D. F. 2003, CQGra, 20, 2045

Barrow, J. D., Sandvik, H. B., \& Magueijo, J. 2002b, PhRvD, 65, 063504

Barrow, J. D., \& Tipler, F. J. 1986, The Anthropic Cosmological Principle (Oxford: Oxford Univ. Press)

Bekenstein, J. D. 1982, PhRvD, 25, 1527

Berengut, J. C., Flambaum, V. V., Ong, A., et al. 2013, PhRvL, 111, 010801

Bleem, L. E., Stalder, B., de Haan, T., et al. 2015, ApJS, 216, 27

Bocquet, S., Dietrich, J. P., Schrabback, T., et al. 2019, ApJ, 878, 55

Bora, K., \& Desai, S. 2021a, JCAP, 02, 012

Bora, K., \& Desai, S. 2021b, JCAP, 2021, 052

Bulbul, E., Chiu, I. N., Mohr, J. J., et al. 2019, ApJ, 871, 50

Carlstrom, J. E., Ade, P. A. R., Aird, K. A., et al. 2011, PASP, 123, 568

Chand, H., Srianand, R., Petitjean, P., \& Aracil, B. 2004, A\&A, 417, 853

Chen, G., \& Ratra, B. 2011, PASP, 123, 1127

Chen, L., Huang, Q.-G., \& Wang, K. 2019, JCAP, 02, 028

Chodos, A., \& Detweiler, S. 1980, PhRvD, 21, 2167

Clara, M. T., \& Martins, C. J. A. P. 2020, A\&A, 633, L11

Colaço, L. R., Gonzalez, J. E., \& Holanda, R. F. L. 2021a, EPJC, 81, 533

Colaço, L. R., Holanda, R. F. L., \& Silva, R. 2021b, EPCJ, 81, 822

Colaço, L. R., Holanda, R. F. L., Silva, R., \& Alcaniz, J. S. 2019, JCAP, 03, 014

Copeland, E. J., Nunes, N. J., \& Pospelov, M. 2004, PhRvD, 69, 023501

Damour, T., \& Dyson, F. 1996, NuPhB, 480, 37

Desai, S., Armstrong, R., Mohr, J. J., et al. 2012, ApJ, 757, 83

Dirac, P. 1937, Natur, 139, 323

Dodelson, S., \& Schmidt, F. 2020, Modern Cosmology (2nd ed.; New York: Academic)

Farajollahi, H., \& Salehi, A. 2012, JCAP, 2012, 041

Foreman-Mackey, D., Hogg, D. W., Lang, D., \& Goodman, J. 2013, PASP, 125,306
Fowler, J. W., Niemack, M. D., Dicker, S. R., et al. 2007, ApOpt, 46, 3444 Galli, S. 2013, PhRvD, 87, 123516

Gamow, G. 1967, PhRvL, 19, 759

Goncalves, R. S., Holanda, R. F. L., \& Alcaniz, J. S. 2012, MNRAS, 420, L43

Hart, L., \& Chluba, J. 2018, MNRAS, 474, 1850

Hees, A., Do, T., Roberts, B. M., et al. 2020, PhRvL, 124, 081101

Hees, A., Minazzoli, O., \& Larena, J. 2014, PhRvD, 90, 124064

Hofmann, F., Sanders, J. S., Clerc, N., et al. 2017, A\&A, 606, A118

Holanda, R. F. L., Busti, V. C., Colaço, L. R., \& Landau, J. S. A. S. J. 2016a, JCAP, 08, 055

Holanda, R. F. L., Colaço, L. R., Gonçalves, R. S., \& Alcaniz, J. S. 2017, PhLB, 767, 188

Holanda, R. F. L., Landau, S. J., Alcaniz, J. S., Sánchez, G. I. E., \& Busti, V. C. 2016b, JCAP, 2016b, 047

Iocco, F., Mangano, G., Miele, G., Pisanti, O., \& Serpico, P. D. 2009, PhR, 472,1

King, J. A., Webb, J. K., Murphy, M. T., et al. 2012, MNRAS, 422, 3370

Kolb, E. W., Perry, M. J., \& Walker, T. P. 1986, PhRvD, 33, 869

Kravtsov, A. V., Vikhlinin, A., \& Nagai, D. 2006, ApJ, 650, 128

Lamoreaux, S. K., \& Torgerson, J. R. 2004, PhRvD, 69, 12170

Mantz, A., Allen, S. W., Ebeling, H., Rapetti, D., \& Drlica-Wagner, A. 2010, MNRAS, 406, 1773

Marciano, W. J. 1984, PhRvL, 52, 489

Marra, V., \& Rosati, F. 2005, JCAP, 5, 011

Martins, C. J. A. P. 2017, RPPh, 80, 126902

Morel, L., Yao, Z., Cladé, P., \& Guellati-Khélifa, S. 2020, Natur, 588, 61

Mosquera, M. E., \& Civitarese, O. 2013, A\&A, 551, A122

Murphy, M., Webb, J., Flambaum, V., et al. 2001a, MNRAS, 327, 1244

Murphy, M., Webb, J., Flambaum, V., et al. 2001b, MNRAS, 327, 1208

Murphy, M., Webb, J., Flambaum, V., Prochaska, J., \& Wolfe, A. 2001c, MNRAS, 327, 1237

Murphy, M. T., Flambaum, V. V., Webb, J. K., et al. 2004, in Lecture Notes in Physics, Astrophysics, Clocks and Fundamental Constants, ed. S. G. Karshenboim \& E. Peik, 648 (Berlin: Springer), 131

Murphy, M. T., Webb, J. K., \& Flambaum, V. V. 2003, MNRAS, 345, 609

Murphy, M. T., Webb, J. K., \& Flambaum, V. V. 2007, PhRvL, 99, 239001

Murphy, M. T., Webb, J. K., \& Flambaum, V. V. 2008, MNRAS, 384, 1053

O’Bryan, J., Smidt, J., Bernardis, F. D., \& Cooray, A. 2015, ApJ, 798, 18

Padmanabhan, T. 2003, PhR, 380, 235

Park, C.-G., \& Ratra, B. 2019, Ap\&SS, 364, 134

Peebles, P. J. E., \& Ratra, B. 1988, ApJ, 325, L17

Piffaretti, R., Arnaud, M., Pratt, G., Pointecouteau, E., \& Melin, J.-B. 2011 A\&A, 534, A109

PLANCK Collaboration 2015, A\&A, 580, A22

Planck Collaboration 2020, A\&A, 641, A6

Samushia, L., \& Ratra, B. 2006, ApJ, 650, L5

Samushia, L., \& Ratra, B. 2009, ApJ, 701, 1373

Sandvik, H. B., Barrow, J. D., \& Magueijo, J. 2002, PhRvL, 88, 031302

Saro, A., Bocquet, S., Rozo, E., et al. 2015, MNRAS, 454, 2305

Song, J., Zenteno, A., Stalder, B., et al. 2012, ApJ, 761, 22

Srianand, R., Chand, H., Petitjean, P., \& Aracil, B. 2004, PhRvL, 92, 121302

Sunyaev, R. A., \& Zel'dovich, Y. B. 1972, CoASP, 4, 173

Teller, E. 1948, PhRv, 73, 801

Uzan, J.-P. 2003, RvMP, 75, 403

Uzan, J.-P. 2011, LRR, 14, 2

Wang, K., \& Chen, L. 2020, EPJC, 80, 570

Webb, J. K., Flambaum, V. V., Churchill, C. W., Drinkwater, M. J., \& Barrow, J. D. 1999, PhRvL, 82, 884

Webb, J. K., King, J. A., Murphy, M. T., et al. 2011, PhRvL, 107, 191101

Webb, J. K., Murphy, M. T., Flambaum, V. V., et al. 2001, PhRvL, 87, 091301 